\title{
Análise fatorial confirmatória da versão portuguesa da escala Preschool Play Behaviour Scale para educadoras
}

\author{
Lígia Monteiro ${ }^{1}$, Marília Fernandes², Tânia Sousa², Nuno Torres², António Rosado ${ }^{3}$ \& \\ António J. Santos ${ }^{2}$ \\ ${ }^{1}$ Instituto Universitário de Lisboa (ISCTE-IUL); CIS-IUL \\ ${ }^{2}$ William James Center for Research, ISPA-Instituto Universitário \\ ${ }^{3}$ Faculdade de Motricidade Humana da Universidade de Lisboa
}

\begin{abstract}
Resumo: A Preschool Play Behavior Scale é um instrumento constituído por 18 itens que expressam diferentes tipos de comportamentos das crianças, em contexto de brincadeira livre com os pares, procurando diferenciar, em particular, tipos de comportamentos não-sociais. 0 presente estudo teve como objetivo traduzir e analisar a estrutura fatorial do instrumento e as suas características psicométricas. Sessenta educadoras descreveram os comportamentos de 369 crianças (166 raparigas), com idades compreendidas entre os 36 e os 60 meses, e a frequentar escolas de Ensino Particular com fins lucrativos e Instituições Particulares de Solidariedade Social, do Distrito de Lisboa/Portugal. 0 modelo final de 4 fatores - Comportamento Reticente, Solitário-Passivo, Solitário-Ativo e Brincadeira Social parece ajustar-se adequadamente aos grupos de idade e sexo considerados.
\end{abstract}

Palavras-chave: Comportamentos Não-sociais; Pré-escolar; Estrutura Fatorial; Educadoras.

Factorial Structure of the PPBS Portuguese Version for Preschool Teachers: The Preschool Play Behaviour Scale (PPBS) is an 18-item questionnaire describing children's behaviours during free play, in the presence of their peers. It aims, mainly, to differentiate different types of non-social behaviours. The goals of this study were to translate and test the factorial structure of the PPBS and its psychometric proprieties. Sixty preschool teachers described the behaviors of 369 children (166 girls), with ages ranging from 36 to 60 months. All attended private pre-school programs in the district of Lisbon/Portugal. A four model factorial structure: Reticent behaviour, Solitary-Passive behaviour, Solitary-Active behaviour, Social Play seems to fit when age and sex groups are considered.

Keywords: Non-Social-Behaviours; Preschool; Factorial Structure; Preschool Teachers.

Nas sociedades contemporâneas, em particular nas ocidentais, as crianças, desde muito cedo, passam várias horas por dia em contexto de Jardim-de-Infância interagindo com adultos e um elevado número de crianças que não fazem parte do seu contexto familiar. Dados de 2014 indicam que as taxas de frequência do pré-escolar (em particular nos 3 e 4 anos) têm vindo a aumentar em Portugal, nos últimos anos, situando-se acima da média da OCDE (OCDE, 2016). De modo a potencializar um ajustamento saudável das crianças ao seu meio sócio/cultural, será fundamental compreender não só o sucesso na iniciação e manutenção de interações positivas com os pares, mas, também, analisar as motivações e características associadas a um reduzido envolvimento social.

É consensual, na área do desenvolvimento, que as interações com os pares são um contributo fundamental para a socialização, criando um importante contexto de aprendizagem social e de aquisição de competências, atitudes e experiências com impacto ao longo da vida (Rubin, Bukowski, \& Laursen, 2009). Nos últimos anos, diversos estudos têm encontrado suporte empírico para a ideia de que crianças que, de um modo consistente, apresentam baixos níveis de "quantidade e qualidade" de interações sociais, durante os primeiros anos de vida, se encontram em risco de desajustamento psicossocial posterior (e.g., Rubin, Bowker, \& Kennedy, 2009; Rubin, Coplan, \& Bowker, 2009).

0 fenómeno da solidão social tem sido definido como um construto que varia em termos de comportamentos, contextos, motivações e consequências para o desenvolvimento (e.g., Rubin, 1982; Rubin \& Asendorpf, 1993; Rubin \& Coplan, 2004; Rubin \& Mills, 1988). No entanto, e pela complexidade do fenómeno, diversos desafios se colocam ao seu estudo, quer ao nível da definição clara dos conceitos quer da sua avaliação. A Preschool Play Behavior Scale (Coplan \& Rubin, 1998) é um instrumento que se

${ }^{1}$ Endereço para correspondência: Lígia Monteiro, Avenida das Forças Armadas (Ala Autónoma, gabinete 101), 1649-026 Lisboa, Portugal. Email: lmsmo@iscte.pt 
inscreve no esforço de diferenciar os vários tipos de comportamentos não-sociais de crianças em idade pré-escolar, como por exemplo: o comportamento Reticente, Solitário-Passivo ou Solitário-Ativo.

A brincadeira não-social é definida como a apresentação consistente (ao longo do tempo e em diferentes contextos) de comportamentos e atividades solitárias na presença de potenciais parceiros de brincadeira; ou seja, face à possibilidade de se envolver em atividades sociais e brincadeiras de grupo, tal não se verifica (Coplan, Ooi, Kirkpatrick, \& Rubin, 2015; Rubin, 1982; Rubin \& Asendorpf, 1993; Rubin \& Mills, 1988).

Diferentes comportamentos não-sociais têm sido descritos na literatura: 0 comportamento Reticente caracteriza-se por a criança se manter frequentemente desocupada durante longos períodos, vagueando pela sala, observando à distância as atividades dos pares, e raramente iniciando contacto com os mesmos (e.g., Coplan, Prakash, O’Neil, \& Armer, 2004; Coplan, Rubin, Fox, Calkins, \& Stewart, 1994). Do ponto de vista motivacional, são crianças que embora possam desejar interagir com os outros, por medo ou timidez, não o fazem (renitência em iniciar a interação) sendo, frequentemente, observadas a brincar sozinhas (e.g., Coplan et al., 2004; Rubin \& Asendorpf, 1993).

Considera-se comportamento Solitário-Passivo quando a criança, de modo não-ansioso, brinca sozinha explorando objetos ou se encontra investida em atividades construtivas (e.g., construção, leitura ou desenho) (Coplan et al., 1994; Rubin, 1982; Rubin, Hymel, \& Mills, 1989). Estes comportamentos parecem ser característicos de crianças que, embora tenham baixa motivação para socializar, não sentem aversão a interagir com os pares, respondendo positivamente quando abordadas ou iniciando interações quando necessário (Asendorpf \& Meier, 1993; Coplan, Girardi, Findlay, \& Frohlick, 2007; Coplan et al., 2004). É, também, designado de Desinteresse Social (Asendorpf, 1990), sendo considerado relativamente benigno, em particular na infância (e.g., Coplan et al., 2004). Contudo, alguns autores (ver Coplan et al., 2015) têm questionado as motivações subjacentes a este tipo de comportamento, indicando que estas podem ser muitos diversas.

O comportamento Solitário-Ativo é caracterizado por ações sensório-motoras repetitivas com ou sem objetos e brincadeiras de dramatização solitárias (e.g., Coplan et al., 1994; Rubin, 1982; Rubin et al., 1989). Estes comportamentos estão associados à imaturidade social e impulsividade, sendo considerados comportamentos não-normativos e percepcionados negativamente pelos pares conduzindo, frequentemente, à rejeição social (e.g., Coplan, Wichmann, \& Lagace-Seguin, 2001; Rubin \& Mills, 1988).

Nos últimos anos, têm sido desenvolvidos diversos tipos de medidas com vista a analisar a brincadeira social e não-social (ver Coplan et al., 2015), baseadas quer na observação direta por parte de observadores treinados, quer na classificação, descrição ou nomeação por parte de professores, pais e/ou pares (considerados como peritos pelo conhecimento que possuem da criança).

\section{A Preschool Play Behavior Scale - PPBS (Coplan \& Rubin, 1998)}

A PPBS é um questionário preenchido pelas educadoras, com base nos comportamentos observados de crianças de idade pré-escolar, no contexto de brincadeira livre. Contrariamente a outras medidas que analisam constructos gerais associados ao funcionamento socioemocional em contexto escolar, a PPBS procura descriminar diferentes tipos de comportamentos não-sociais: (1) comportamento Reticente, (2) Solitário-Passivo e (3) Solitário-Ativo, no contexto de sala de aula (Coplan \& Rubin, 1998). Os comportamentos de brincadeira social são, também, contemplados no instrumento, e referem-se a comportamentos de brincadeira que ocorrem no contexto das interações ao nível diádico ou de grupo. São considerados os comportamentos de Brincadeira Social (e.g., conversas entre crianças, brincadeiras dramáticas e de grupo) e de Brincadeira de Lutas (e.g., brincadeiras mais físicas e de luta faz-de-conta). Deste modo, as educadoras têm um leque mais vasto e diversificado de comportamentos para avaliar, possibilitando, ainda, a analise da validade divergente em relação aos comportamentos não-sociais (Coplan \& Rubin, 1998).

Segundo Coplan et al. (2015), diversas são as vantagens associadas à utilização de educadores/professores como informantes do tipo ou frequência dos comportamentos das crianças. Estes têm a possibilidade de observar os seus comportamentos em diversos contextos e situações do dia a dia e, não fazendo parte do grupo de pares, poderão descrever características ou comportamentos das crianças de modo mais objetivo. Estas recolhas são ainda, e por norma, pouco dispendiosas em termos de tempo e recursos humanos, comparativamente com as medidas de observação.

No sentido de fundamentar a validade de construto da PPBS, Coplan e Rubin (1998) verificaram que as descrições das educadoras e a observação direta dos diversos tipos de comportamentos, nãosociais e sociais, se encontravam significativamente associadas. Confirmaram-se as propriedades psicométricas (e.g., estrutura fatorial, consistência interna, validade convergente e discriminante) do instrumento utilizando, ainda, medidas respondidas por mães e educadores. Deste modo, na perspetiva 
dos autores, a PPBS constitui-se como uma potencial mais valia, a ser utilizada em parceria com medidas de observação ou mesmo em sua substituição (Coplan \& Rubin, 1998).

Sendo um instrumento desenvolvido e utilizado em amostras anglo-saxónicas já foi, no entanto, traduzido e adaptado para outras culturas. Numa amostra de 141 crianças da Malásia, Choo, Xu e Haron (2012) realizaram uma análise fatorial confirmatória, tendo verificado que as educadoras distinguiam os três subtipos de comportamentos não-sociais, em crianças de idade pré-escolar. Verificaram, ainda, que esta estrutura se aplica de modo semelhante a raparigas e rapazes. Igualmente, numa amostra $(n=1622)$ de baixo rendimento económico, originária de Hong Kong, o modelo de cinco fatores da PPBS adequou-se aos dados, embora o número total de itens tenha sido reduzido para 14, dado 4 terem sido identificados como redundantes (Leung, 2015).

Os objetivos do presente estudo foram traduzir o questionário Preschool Play Behavior Scale para Português; testar a estrutura fatorial proposta por Coplan e Rubin (1998) e avaliar as características psicométricas do mesmo.

\section{MÉTODO}

\section{Participantes}

Participaram no estudo 60 educadoras que descreveram os comportamentos de 369 crianças (166 raparigas e 203 rapazes), com idades compreendidas entre os 36 e os 60 meses $(M=50.88$; $D P=6.53)$. Os dados foram organizados em 2 grupos de idade, 130 crianças (50 raparigas; 80 rapazes) de 4 anos $(M=$ $43.27 ; D P=3.20$ ) e 239 crianças (116 raparigas; 123 rapazes) de 5 anos $(M=55.02 ; D P=3.39) .45 \%$ das crianças frequentavam Instituições Particulares de Solidariedade Social e as restantes Escolas Particulares com fins lucrativos, em salas de pré-escolar organizadas em função das suas idades, e constituídas por 15 a 25 crianças. A maioria das educadoras (96\%) possuía licenciatura/mestrado e as restantes o bacharelato. As suas idades variavam entre os 26 e os 60 anos $(M=39.71 ; D P=9.20)$, tendo em média $15.48(D P=10.50)$ anos de serviço.

As crianças viviam com ambos os pais (famílias nucleares), tendo as mães idades compreendidas entre os 22 e os 50 anos $(M=35.13 ; D P=5.18)$ e os pais entre os 19 e os 59 anos $(M=37.68 ; D P=6.08)$. As habilitações literárias maternas variavam entre os 6 e os 21 anos de escolaridade $(M=14.36$; $D P=$ $3.32)$ e as paternas entre os 4 e os 19 anos $(M=12.81$; $D P=3.50)$. $82.4 \%$ das mães e $89.6 \%$ dos pais trabalhavam (tempo inteiro), em média $39.03(D P=6.78)$ e $41.78(D P=5.89)$ respetivamente. As famílias viviam no Distrito de Lisboa, Portugal. Esta é uma amostra de conveniência.

\section{Instrumentos}

Medidas sociodemográficas

A Ficha de Identificação (Veríssimo, não publicada) visa recolher informação relativa aos dados sociodemográficos da família (figuras parentais, criança e agregado familiar).

\section{Preschool Play Behavior Scale}

A Preschool Play Behavior Scale (Coplan \& Rubin, 1998) é um instrumento constituído por 18 itens que descrevem comportamentos de crianças em idade pré-escolar que podem ser observados em contexto de brincadeira livre com os pares. É composto por três dimensões que remetem para diferentes tipos de comportamentos não-sociais: (1) Comportamento Reticente constituída por quatro itens que remetem para comportamentos como o observar os pares sem interagir, ou o estar desocupado (e.g., "Vagueia pela sala sem qualquer objectivo"); (2) Comportamento Solitário-Passivo constituída por quatro itens relacionados com comportamentos solitários construtivos e de exploração (e.g., "Brinca sozinho, explorando brinquedos ou objetos, tentando perceber como eles funcionam"); (3) Comportamento Solitário-Ativo constituída por 2 itens que remetem para comportamentos solitários dramáticos (e.g., "Brinca ao faz-de-conta, mas sozinho"). Existem, ainda, duas dimensões que permitem abranger uma maior diversidade de comportamentos de brincadeira: (4) Brincadeira Social constituída por seis itens relacionados com a brincadeira em grupo, brincadeira sócio-dramática e conversa com os pares (e.g., "Conversa com outras crianças enquanto brinca"); e (5) Brincadeira de Lutas ("rough and tumble play") constituída por 2 itens consiste em comportamentos de brincadeira física, assim como lutas a fingir (e.g., "Envolve-se em lutas simuladas e prazerosas/divertidas com outras crianças"). As educadoras respondem, ao questionário, numa escala de Likert de 5 pontos: 1 - Nunca; 2 - Quase nunca; 3 - Às vezes; 4 - Frequentemente; 5 - Muito frequentemente. 


\section{Procedimento}

Numa fase inicial, fez-se a tradução da versão original do instrumento para Português, obedecendo aos critérios referenciados para adaptação transcultural de questionários psicológicos de Brislin (1980), designada de "abordagem por comité" (committee approach). Uma primeira versão foi aplicada a um pequeno grupo de educadores para pré-teste qualitativo, de forma a garantir que todos os itens eram compreensíveis.

Esta recolha foi realizada no âmbito de um projeto mais vasto, aprovado pela Comissão de Ética do ISCTE-IUL. Todos os pais assinaram um consentimento para a participação dos filhos no estudo. As educadoras responderam ao questionário apenas em relação às crianças autorizadas, sendo que o número de sujeitos descritos por cada educadora foi em média de $6.15(D P=3.90)$. Foi-lhes pedido que preenchessem a PPBS de acordo com a frequência com que observam o comportamento descrito (em cada item) nos momentos de brincadeira livre de cada criança alvo. Os questionários foram preenchidos no início do 3. ${ }^{\circ}$ período escolar, de modo a garantir que as educadoras estivessem bem familiarizadas com todas as crianças da sala. Para a cotação do questionário, é calculada uma média aritmética para cada dimensão.

\section{Análise de Dados}

De modo a testar a replicabilidade do modelo de cinco fatores proposto pelos autores do questionário na sua versão original (Coplan \& Rubin, 1998), recorreu-se a uma análise fatorial confirmatória (AFC) com o software AMOS (v.21, SPSS Inc, Chicago, IL) através do método da Máxima Verosimilhança (ML).

Num primeiro momento, analisou-se a normalidade univariada e multivariada da distribuição dos valores das variáveis, através dos coeficientes de assimetria $(S k, \operatorname{com}|S k|<3)$, de curtose $(K u, \operatorname{com}|K u|<$ 10) e do coeficiente de Mardia. Testou-se, também, a presença de outliers através da distância quadrada de Mahalanobis $\left(D^{2}\right.$ ) (Kline, 2005). Posteriormente, e de modo a avaliar a qualidade do ajustamento do modelo proposto, utilizaram-se os índices definidos por Hu e Bentler (1999) e os valores de $\chi 2$. Este último apresenta limitações consoante o tamanho da amostra, da complexidade do modelo, ou quando os pressupostos de normalidade não são validados (Cheung \& Rensvold, 2002). Assim, considera-se que o modelo apresenta um bom ajustamento quando os valores de CFI (Comparative Fit Index) são superiores .95; os valores de SRMR (Standardized Root Mean Squared Residual) são menores que .08, e os valores de RMSEA (Root Mean Square Error of Approximation) são menores que .06. São, ainda, considerados aceitáveis valores CFI superiores a .90, e valores de SRMR e RMSEA até .10 (Hu \& Bentler, 1999). Foram, também, utilizados índices que permitem a comparação entre modelos: Akaike Information Criterion (AIC) e Modified Expected Cross-Validation Index (MECVI), nestes casos, quanto menor o valor apresentado, melhor o ajustamento do modelo (Byrne, 2001). Para analisar a adequação do modelo nos diferentes grupos considerados (sexo e idade), testou-se a invariância configuracional (que avalia a adequação da estrutura dos constructos latentes), a invariância métrica (que testa a invariância dos pesos fatoriais entre grupos, comparando o modelo com os pesos fatoriais livres e o modelo com pesos fatoriais quando restringidos à igualdade entre grupos), e a invariância escalar do modelo (avalia a invariância das médias entre os grupos, comparando com o modelo em que tanto os pesos fatoriais, como as médias fatoriais estão restringidas de modo a serem iguais entre os grupos) (Meredith, 1993). Mais uma vez, tendo em consideração as limitações do $\chi^{2}$, utilizaram-se os valores de referência propostos por Chen (2007). Deste modo, considerou-se invariância dos pesos fatoriais quando as diferenças do CFI entre os modelos $(\triangle \mathrm{CFI}$ ) é menor que .01, a diferença do RMSEA ( $\triangle$ RMSEA) menor que .015 e a diferença do SRMS $(\triangle \mathrm{SRMR})$ menor que .03. Relativamente à invariância das médias fatoriais, são considerados valores de $\triangle$ CFI, $\triangle$ RMSEA e $\triangle$ SRMR menores que $.01, .015$ e .01 respetivamente (Chen, 2007).

Por fim, procedeu-se à avaliação da qualidade do ajustamento local do modelo final, nomeadamente, à avaliação da validade convergente e discriminante, bem como da fiabilidade individual $\left(\lambda^{2}\right)$ e compósita dos itens (FC). A validade convergente foi avaliada através do cálculo da variância média extraída (VEM) de cada fator (Fornell \& Larcker, 1981; Hair, Black, Babin, \& Anderson, 2010). As VEM foram comparadas com o quadrado das correlações entre fatores de modo a obter uma estimativa dos valores da validade discriminante (Fornell \& Larcker, 1981).

\section{RESULTADOS}

Na tabela 1 são apresentadas as médias e os desvios-padrão das escalas da PPBS. Na análise preliminar, foram identificados 4 outliers multivariados, a sua remoção não conduziu a melhorias no modelo inicial, pelo que os sujeitos foram novamente incluídos. 
Tabela 1. Média e Desvio Padrão para as Dimensões da PPBS

\begin{tabular}{lccccc}
\hline & \multicolumn{5}{c}{$\boldsymbol{M}(\boldsymbol{S D})$} \\
\cline { 2 - 6 } & Geral & Raparigas & Rapazes & $\mathbf{4}$ anos & $\mathbf{5}$ anos \\
\hline Comportamento Reticente & $2.33(.77)$ & $2.34(.81)$ & $2.32(.75)$ & $2.44(.78)$ & $2.27(.77)$ \\
Comportamento Solitário-Passivo & $3.17(.71)$ & $3.08(.75)$ & $3.24(.67)$ & $3.21(.74)$ & $3.15(.70)$ \\
Comportamento Solitário-Ativo & $2.81(.91)$ & $2.80(.99)$ & $2.81(.84)$ & $2.92(1.01)$ & $2.75(.84)$ \\
Brincadeira Social & $3.95(.77)$ & $3.97(.80)$ & $3.94(.76)$ & $3.88(.85)$ & $3.99(.72)$ \\
Brincadeira de Luta & $2.46(1.27)$ & $1.66(.81)$ & $3.08(1.20)$ & $2.43(1.24)$ & $2.47(1.28)$ \\
\hline
\end{tabular}

As variáveis apresentam normalidade univariada (Kline, 2005), com todos os valores de $S k$ e $K u$ menores ou iguais a 1 . No que respeita à normalidade multivariada, o coeficiente de Mardia apresentou um valor de 34.96. Como alternativa, poderia ser utilizado o método da distribuição assimptótica livre (ADF) que não exige normalidade multivariada, no entanto, este requer amostras de grandes dimensões (Hu, Bentler, \& Kano, 1992), por outro lado, tendo em conta o valor elevado do coeficiente de Mardia, também, o método de Bootstrap parece ser menos vantajoso que o método ML inicialmente utilizado (Ory \& Mokhtarian, 2010). Por estas razões, manteve-se a análise através do método de ML.

De seguida, testou-se o ajustamento do modelo original da PPBS à amostra de 369 questionários. 0 resultado obtido foi pouco satisfatório $\left\{\chi^{2}(369)=610.16 ; \chi^{2} / \mathrm{df}=4.88\right.$; CFI = .87; RMSEA = .10; SRMR= .07; $\mathrm{AIC}=702.16$; MECVI = 1.92\} considerando os valores referidos na literatura (ver Byrne, 2001). De modo a melhorar o ajustamento do modelo, e com base em considerações teóricas, foram incluídas trajetórias entre os resíduos de pares de itens pertencentes ao mesmo fator, cujos valores de índices de modificação pelos multiplicadores de Lagrange (LM) eram superiores a 11 ( $p<.001$ ) (Hair et al., 2010). Assim, os seguintes pares de itens parecem partilhar o mesmo conteúdo pelo que procedemos à covariação dos seus erros (Kline, 2005): Itens 4 e 12 do Comportamento Reticiente, ambos os itens remetem para o papel de espectador da criança na interação com os outros; Itens 1 e 15 da Brincadeira Social, ambos remetem para o conversar com outras crianças enquanto brinca. Foram removidos os itens associados a outros fatores que não os previstos no modelo original: Item 5, originalmente no fator Brincadeira Social, surge associado aos fatores Comportamento Solitário-Passivo e Comportamento Solitário-Ativo; Item 9, originalmente no fator Comportamento Reticiente, surge associado ao fator Brincadeira de Luta; Item 10, originalmente no fator Brincadeira Social, mas que surge associado aos fatores Comportamento Solitário-Passivo e Comportamento Solitário-Ativo; Item 17, originalmente no fator comportamento Solitário-Passivo, mas que surge associado ao fator Brincadeira Social). Desta forma, o modelo de cinco fatores (ver Figura 1 ) apresenta um bom ajustamento $\left\{\chi^{2} / \mathrm{df}=3.02\right.$; CFI $=.95$; RMSEA = $.07 ; \mathrm{P}[\mathrm{rmsea}]=.000 ; \mathrm{SRMR}=.05 ; \mathrm{AIC}=276.26 ; \mathrm{MECVI}=.76\}$.

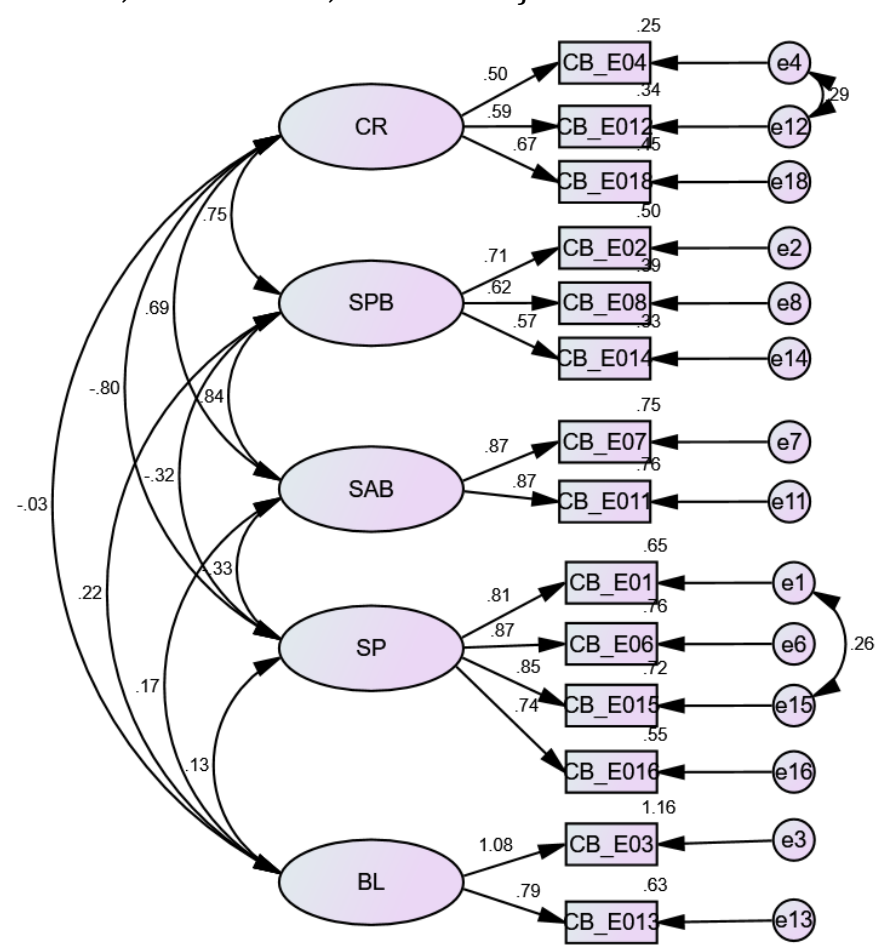

Note. CR-Comp. Reticente; CSP - Comp. Solitário-Passivo; CSA - Comp. Solitário-Ativo; BS-Brincadeira Social; BL-Brincadeira de Lutas

Figura 1. Modelo cinco fatores da Preschool Play Behaviour Scale. 


\section{Invariância do modelo considerando o sexo}

De seguida, testou-se o ajustamento fatorial do modelo obtido anteriormente, a ambos os sexos. 0 modelo das raparigas obteve os seguintes valores $\chi^{2} / \mathrm{df}=2.08$; $\mathrm{CFI}=.94$; RMSEA $=.08$; $\mathrm{P}[\mathrm{rmsea}]=.005$; SRMR= $.05 ; \mathrm{AIC}=215.28$; MECVI $=1.38\}$, por sua vez, o modelo dos rapazes $\left\{\chi^{2} / \mathrm{df}=2.21 ; \mathrm{CFI}=.94 ; \mathrm{RMSEA}=.08\right.$; $\mathrm{P}[\mathrm{rmsea}]=.005 ; \mathrm{SRMR}=.06$; $\mathrm{AIC}=223.79 ; \mathrm{MECVI}=1.14\}$, e os valores obtidos na análise multigrupos foram os seguintes $\left\{\chi^{2} / \mathrm{df}=2.15\right.$; CFI = .94; RMSEA = .06; $\mathrm{P}[\mathrm{rmsea}]=.13$; $\mathrm{SRMR}=.05$; $\mathrm{AIC}=439.08$; $\mathrm{MECVI}$ $=1.25\}$. Tendo em consideração os resultados, pode-se admitir a invariância configuracional do modelo, querendo isto dizer que os construtos implicados se adequam a ambos sexos.

Relativamente à invariância métrica, isto é, quando comparamos o modelo com os pesos fatoriais livres e o modelo com pesos fatoriais restringidos à igualdade entre os grupos, obtivemos os seguintes valores $\Delta \chi^{2}(9)=14.95 \mathrm{com} p=.09 ; \Delta \mathrm{CFI}=.002 ; \Delta \mathrm{RMSEA}=.001 ; \Delta \mathrm{SRMS}=.003$. Deste modo, tendo em consideração os valores de referência apresentados por Chen (2007), os pesos fatoriais são equivalentes entre raparigas e rapazes. No que respeita à invariância escalar, isto é, quando analisámos se existem diferenças na qualidade do modelo quando, também, as médias entre grupos estão restringidas à igualdade, obtivemos os seguintes valores $\Delta \chi^{2}(14)=187.84 \operatorname{com} p<.001 ; \Delta$ CFI $=.07 ; \Delta$ RMSEA $=.022$; $\Delta$ SRMS $=.0013$. Assim, parecem existir diferenças a considerar. Os principais itens responsáveis são os do fator Brincadeira de Luta (item 3 - com 1.70 de média para as raparigas e 3.02 de média para os rapazes, $\mathrm{Z}=11.96 \mathrm{p}<.01 \mathrm{e}$ item 13 - com 1.61 de média para as raparigas e 3.13 de média para os rapazes com $\mathrm{Z}=$ $13.46 \mathrm{p}<.01)$. Quando retirados estes itens, os valores passaram a ser $\Delta \chi^{2}(12)=38.28$ com $p<.001$; $\Delta \mathrm{CFI}=.013 ; \Delta \mathrm{RMSEA}=.003 ; \Delta \mathrm{SRMS}=.0004$, indicando que o modelo parece ser equivalente entre rapazes e raparigas, quando se considera esta alteração. Deste modo, o modelo final de quatro fatores apresenta um bom ajustamento $\left\{\chi^{2} / \mathrm{df}=2.70 ; \mathrm{CFI}=.96\right.$; $\mathrm{RMSEA}=.07 ; \mathrm{P}[\mathrm{rmsea}]=.02$; $\mathrm{SRMR}=.05 ; \mathrm{AIC}=188.34 ; \mathrm{MECVI}$ $=.52\}$.

\section{Invariância do modelo considerando o grupo de idade}

Testou-se, de seguida, o ajustamento fatorial do modelo obtido anteriormente aos dois grupos de idade. 0 modelo dos 4 anos obteve os seguintes valores $\chi^{2} / \mathrm{df}=2.27$; CFI $=.93$; RMSEA $=.10 ; \mathrm{P}[\mathrm{rmsea}]=.001$; $\mathrm{SRMR}=.09 ; \mathrm{AIC}=168.60 ; \mathrm{MECVI}=1.36\}$. Por sua vez, o modelo dos 5 anos $\left\{\chi^{2} / \mathrm{df}=1.83\right.$; CFI = .97; RMSEA $=.06 ; \mathrm{P}[\mathrm{rmsea}]=.22 ; \mathrm{SRMR}=.04 ; \mathrm{AIC}=148.19 ; \mathrm{MECVI}=.64\}$. Os valores obtidos na análise multigrupos foram os seguintes $\left\{\chi^{2} / \mathrm{df}=2.05\right.$; CFI $=.95$; RMSEA $=.05$; P $[$ rmsea $]=.28$; SRMR $=.09$; AIC = 315.92; MECVI $=.89\}$. Face aos resultados obtidos, pode admitir-se a invariância configuracional do modelo, ou seja, os construtos implicados adequam-se a ambas as idades.

No que respeita à invariância métrica, obtivemos os seguintes valores $\Delta \chi^{2}(8)=24.01$ com $p=.22$; $\Delta \mathrm{CFI}=.001 ; \Delta \mathrm{RMSEA}=.002 ; \Delta \mathrm{SRMS}=.004$. Deste modo, tendo em consideração os valores de referência apresentados por Chen (2007), os pesos fatoriais são equivalentes entre o grupo dos 4 e dos 5 anos. Para a invariância escalar obtivemos os seguintes valores $\Delta \chi^{2}(12)=29.14$ com $p<.004 ; \quad \Delta$ CFI=.008; $\triangle$ RMSEA=.001; $\Delta$ SRMS=.0006; também a este nível os modelos de 4 e 5 anos parecem ser invariantes.

Na Tabela 2 são apresentadas informações complementares do modelo final, nomeadamente pesos fatoriais estandardizados $(\lambda)$, fiabilidade individual dos itens $\left(\lambda^{2}\right)$, fiabilidade compósita dos fatores (FC) e a variância extraída média (VEM). A grande maioria dos itens apresentam pesos fatoriais estandardizados superiores a .5, exceto o item 4 (do Comportamento Reticente) que apresenta o valor .49. As dimensões Comportamento Solitário-Ativo e Brincadeira Social, revelaram elevada fiabilidade compósita e validade convergente, sendo a dimensão de Comportamento Reticente (VEM=.34) a que apresenta o desempenho mais frágil. Os itens deste fator poderão estar a correlacionar-se com outros fatores, originando alguns problemas ao nível da validade discriminante. 
Tabela 2. Pesos Fatoriais Estandardizados e Fiabilidade Individual dos Itens, Fiabilidade Compósita e Variância Extraída Média (VEM) das Dimensões da PPBS

\begin{tabular}{|c|c|c|c|c|c|}
\hline Dimensão & Item & $\lambda$ & $\lambda^{2}$ & FC & VEM \\
\hline \multirow[t]{3}{*}{ Comportamento Reticente } & 4 & .49 & .24 & .61 & .34 \\
\hline & 12 & .59 & .34 & & \\
\hline & 18 & .67 & .45 & & \\
\hline \multirow[t]{3}{*}{ Comportamento Solitário-Passivo } & 2 & .70 & .48 & .67 & .40 \\
\hline & 8 & .61 & .37 & & \\
\hline & 14 & .60 & .36 & & \\
\hline \multirow[t]{2}{*}{ Comportamento Solitário-Ativo } & 7 & .86 & .74 & .90 & .69 \\
\hline & 11 & .87 & .76 & & \\
\hline \multirow[t]{4}{*}{ Brincadeira Social } & 1 & .81 & .65 & .86 & .75 \\
\hline & 6 & .87 & .76 & & \\
\hline & 15 & .85 & .72 & & \\
\hline & 16 & .74 & .65 & & \\
\hline
\end{tabular}

Nota $\boldsymbol{\lambda}=$ Peso Fatorial Estandardizado; $\lambda^{2}=$ Fiabilidade Individual do Item; FC = Fiabilidade Compósita; VEM = Variância Extraída Média

Considerando algumas limitações ao nível da validade convergente e discriminante, o modelo final de quatro fatores (ver Figura 2) parece ajustar-se adequadamente aos grupos de idade e sexo considerados.

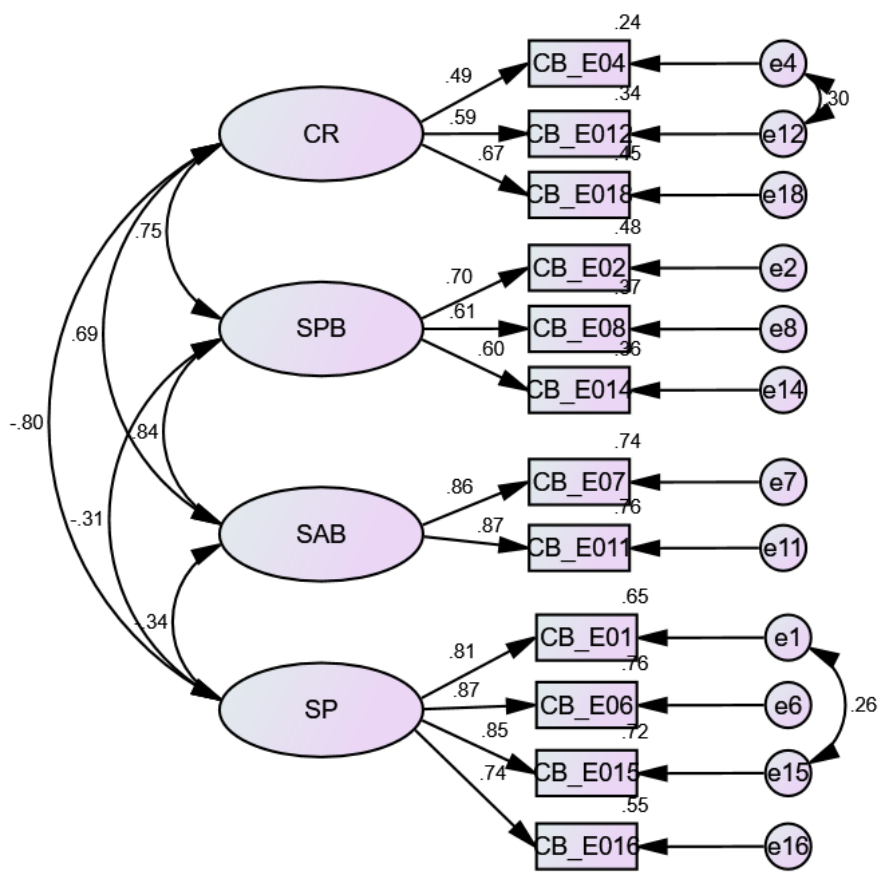

Note. CR-Comp. Reticente; CSP - Comp. Solitário-Passivo; CSA - Comp. Solitário-Ativo; BS-Brincadeira Social; BL-Brincadeira de Lutas

Figura 2. Modelo de quatro fatores da Preschool Play Behaviour Scale.

\section{DISCUSSÃo}

O presente trabalho propôs-se confirmar, numa amostra portuguesa de crianças em idade pré-escolar, a estrutura fatorial definida por Coplan e Rubin (1998) da Preschool Play Behaviour Scale. Para tal, realizouse uma análise fatorial confirmatória no contexto dos modelos de equações estruturais, dos dezoito itens da escala original. Quatro foram retirados, dado que estavam associados a mais do que um fator, tendo o mesmo sucedido noutras adaptações internacionais (ver Choo et al., 2012; Leung, 2015). A estrutura de cinco fatores original foi replicada com índices de ajustamento que sustentam a boa qualidade do modelo.

De modo geral, verifica-se que as educadoras discriminam os comportamentos sociais, dos comportamentos não-sociais. A dimensão Comportamento Reticente que remete para comportamentos em que a criança de modo passivo apenas observa as outras sem participar, parece ter sido a menos compreendida pelas educadoras, apresentando problemas ao nível da fiabilidade compósita, da validade 
convergente e discriminante. 0 item 4 - "Assume o papel de espectador" apresentou pouco fiabilidade individual, o mesmo se tendo verificado no estudo de Leung (2015). Mantiveram-se três itens (dos quatro originais) que remetem para comportamentos em que a criança permanece desocupada ou observa as outras crianças sem se juntar a estas. 0 item 9 - "Vagueia pela sala sem objetivo" foi retirado uma vez que na amostra em estudo se encontrava associado à dimensão Comportamento de Luta, que pode ser considerada uma forma particular de brincadeira social (Pellis, Pellis, \& Bell 2010).

No que se refere à dimensão Solitário-Passivo, mantiveram-se três itens (dos quatro originais) que remetem para comportamentos solitários de exploração de objetos ou de atividades construtivas. 0 item 17 - "Brinca sozinho(a) a explorar objetos ou brinquedos tentando perceber como é que estes funcionam" foi retirado, dado estar associado à dimensão da Brincadeira Social. No estudo de Leung (2015) este item foi mantido, apesar de apresentar uma baixa fiabilidade individual. Este resultado parece ir ao encontro da sugestão de Rubin (1982) de que, nestas idades, as educadoras poderão encorajar este tipo de comportamentos e, portanto, considerá-los como sociais. Por outro lado, apesar dos comportamentos solitários-passivos estarem associados a níveis mais baixos de iniciação de interação e a um desinteresse social, não parecerem estar concorrentemente associados a índices de desajustamento (ver Rubin, Coplan, et al., 2009).

A dimensão do comportamento Solitário-Ativo manteve os items originais da escala. Embora possam ser pouco observados durante os momentos de brincadeira livre (cerca de $3 \%$ do tempo total), quando ocorrem são bastante salientes para pares e educadoras (Rubin, 1982).

Os comportamentos da dimensão Brincadeira Social, que refletem a capacidade da criança para brincar e participar ativamente em conversas e interações construtivas com outros, são descritos pelas educadoras como ocorrendo com maior frequência. Tal resultado seria expectável, dado o período de desenvolvimento em questão, e se tratar de uma amostra normativa, sem risco identificado. Foram retirados dois itens, ficando esta dimensão com quatro itens (face aos seis originais), à semelhança de Leung (2015): o item 5 - relacionado com o brincar ao imaginário com outras crianças, que surge associado às dimensões de comportamento solitário, assim como, o item 10 - "Brinca em grupo com com outras crianças (não apenas ao lado de)".

Outra forma de brincadeira são os designados comportamentos de luta a fingir, mais físicas, de natureza estimulante e desafiadora que, naturalmente, pode potencializar o surgimento de conflitos entre pares, pela sua natureza, e implicar algum "scaffolding" por parte das educadoras. É nesta dimensão, e para esta amostra, que parecem residir as diferenças de sexo. Quando se testa o modelo para rapazes e raparigas, verificamos que o valor de ajustamento é bom para ambos, relevando-se equivalentes ao nível da invariância métrica. No entanto, quando consideramos o nível da invariância escalar, isto é, em que medida as médias dos grupos poderão ser diferentes, a dimensão Brincadeira de Luta destaca-se, com os rapazes a apresentarem valores mais elevados que as raparigas. No estudo original de Coplan \& Rubin (1998) a estrutura factorial da escala reportada para rapazes e raparigas é semelhante e consistente com os resultados da amostra total. Embora os rapazes sejam descritos pelas educadoras como apresentando valores significativamente superiores neste tipo de comportamentos.

Os resultados obtidos sugerem a aplicabilidade do modelo de cinco fatores propostos por Coplan e Rubin (1998). No entanto, quando se pretende comparar os grupos de rapazes e raparigas são necessários alguns cuidados, nomeadamente, na dimensão que remente para os comportamentos de brincadeiras mais físicas, duras e de lutas a fingir. Gostaríamos, ainda, de salientar que os resultados obtidos deverão ser replicados com outras metodologias, nomeadamente de observação dos comportamentos, em contexto natural, por observadores treinados. As educadoras poderão não estar a identificar e interpretar alguns comportamentos adequadamente (Coplan, Rubin, \& Findlay, 2006), em particular, em grupos cujo o rácio educador/criança é elevado. Em Portugal, este é em média de 16.6, um dos mais elevados dos países da OCDE, de acordo com o relatório Education at Glance (2016). Relativamente às características socioeconómicas e educativas das famílias será interessante obter uma maior diversidade, assim como testar o instrumento em amostras com diferentes níveis de risco, onde os comportamentos não sociais possam ser mais salientes. Deverá, ainda, ser testada a validade por referência ao critério (concorrente e preditiva). No entanto, os resultados obtidos são um primeiro passo para a utilização da PPBS no domínio da investigação em contexto natural, e na tentativa de identificação precoce de potenciais dificuldades de interação em contexto pré-escolar.

\section{Referências}

Asendorpf, J. (1990). Beyond social withdrawal: Shyness, unsociability, and peer avoidance. Human Development, 33, 250-259. https://doi.org/10.1159/000276522 
Asendorpf, J. B., \& Meier, G. H. (1993). Personality effects on children's speech in everyday life: Sociabilitymediated exposure and shyness-mediated reactivity to social situations. Journal of Personality and Social Psychology, 64, 1072-1083. https://doi.org/10.1037/0022-3514.64.6.1072

Brislin, R.W. (1980). Translation and content analysis of oral and written material. In H. C. Triandis \& J. W. Berry (Eds.), Handbook of Cross-Cultural Psychology, (vol. 2, pp. 389-444). Boston, MA: Allyn \& Bacon.

Byrne, B. M. (2001). Structural equation modeling with AMOS: Basic concepts, applications and programming. Mahwah, NJ: Erlbaum.

Chen, F. F. (2007). Sensitivity of Goodness-of-Fit Indexes to Lack of Measurement Invariance. Structural Equation Modeling, 14(3), 464-504. https://doi.org/10.1080/10705510701301834

Cheung, G. W. \& Rensvold, R. B. (2002). Evaluating Goodness-of-Fit Indexes for Testing Measurement $\begin{array}{llll}\text { Invariance. Structural Equation } & \text { Modeling, 9(2), 233-255. }\end{array}$ https://doi.org/10.1207/S15328007SEM0902_5

Choo, M. S., Xu, Y., \& Haron, P. F. (2012). Subtypes of nonsocial play and psychosocial adjustment in Malaysian preschool children. Social Development, 21(2), 294-312. https://doi.org/10.1111/j.1467-9507.2011.00630.x

Coplan, R. J., \& Rubin, K. H. (1998). Exploring and assessing non-social play in the preschool: The development and validation of the preschool play behavior scale. Social Development, 7, 72-91. https://doi.org/10.1111/1467-9507.00052

Coplan, R. J., Girardi, A., Findlay, L. C., \& Frohlick, S. L. (2007). Understanding solitude: Young children's attitudes and responses toward hypothetical socially withdrawn peers. Social Development, 16, 390-409. https://doi.org/10.1111/j.1467-9507.2007.00390.x

Coplan, R. J., Ooi, L., Kirkpatrick, A., \& Rubin, K. H. (2015). Social and nonsocial play. In D. P. Fromberg \& D. Bergen (Eds.), Play from birth to twelve: Contexts, perspectives, and meanings (pp. 97-106). New York, NY: Routledge.

Coplan, R. J., Prakash, K., O'Neil, K., \& Armer, M. (2004). Do you "want" to play? Distinguishing between conflicted shyness and social disinterest in early childhood. Developmental Psychology, 40(2), 244258. https://doi.org/10.1037/0012-1649.40.2.244

Coplan, R. J., Rubin, K. H., \& Findlay, L. C. (2006). Social and nonsocial play. In D.P. Fromberg \& D. Bergen (Eds.), Play from birth to twelve: Contexts, perspectives, and meanings (pp. 75-86) New York, NY: Garland.

Coplan, R. J., Rubin, K. H., Fox, N. A., Calkins, S. D., \& Stewart, S. L. (1994). Being alone, playing alone, and acting alone: Distinguishing among reticence, and passive, and active-solitude in young children. Child Development, 65, 129-138. https://doi.org/10.2307/1131370

Coplan, R. J., Wichmann, C., \& Lagace-Seguin, D. (2001). Solitary active play: A marker variable for maladjustment in preschool? Journal of Research in Childhood Education, 15, 164-172. https://doi.org/10.1080/02568540109594957

Fornell, C., \& Larcker, D. F. (1981). Evaluating Structural Equation Models with Unobservable Variables and Measurement Error. Journal of Marketing Research, 18, 39-50. https://doi.org/10.2307/3151312

Hair, J., Black, W. C., Babin, B. J., \& Anderson, R. E. (2010). Multivariate data analysis (7th ed.). Upper saddle River, NJ: Pearson Education International.

Hu, L., Bentler, P. M., \& Kano, Y. (1992). Can test statistics in covariance structure analysis be trusted? Psychological Bulletin, 112, 351-362. https://doi.org/10.1037//0033-2909.112.2.351

Hu, L.T. and Bentler, P.M. (1999), "Cutoff Criteria for Fit Indexes in Covariance Structure Analysis: Conventional Criteria versus New Alternatives," Structural Equation Modeling, 6 (1), 1-55. https://doi.org/10.1080/10705519909540118

Kline, R. B. (2005). Principles and practice of structural equation modeling (2nd Edition ed.). New York, NY: The Guilford Press.

Leung, C. H. (2015). Factor structure of PPBS with Chinese preschoolers from low-income families. Children and Youth Services Review, 53, 157-165. https://doi.org/10.1016/j.childyouth.2015.04.002

Meredith, W. (1993). Measurement Invariance, Factor Analysis and Factorial Invariance. Psychometrika 58(4):525-543. https://doi.org/10.1007/BF02294825

OCDE (2016). Education at Glace. Retrived from http://www.keepeek.com/Digital-AssetManagement/oecd/education/education-at-a-glance-2016_eag-2016-en\#.WOFPQ0ssocc\#page300

Ory, D.T. \& Mokhtarian, P.L. (2010). The impact of non-normality, sample size and estimation technique on goodness-of-fit measures in structural equation modeling: evidence from ten empirical models 
of travel behavior. Quality \& Quantity, 44, (3), 427-445. https://doi.org/10.1007/s11135-0089215-6

Pellis, S.M., Pellis, V.C., \& Bell, H.C. (2010). The functions of play in the development of the social brain. American Journal of Play, 2, 278-296. https://doi.org/10.1111/j.1467-8721.2007.00483.x

Rubin, K. H., \& Asendorpf, J. (1993). Social withdrawal, inhibition, and shyness in childhood. Hillsdale, NJ: Erlbaum Associates.

Rubin, K. H., \& Coplan, R. J. (2004). Paying attention to and not neglecting social withdrawal and social isolation. Merrill-Palmer Quarterly, 50, 506-534. https://doi.org/10.1353/mpq.2004.0036

Rubin, K. H., \& Mills, R. S. (1988). The many faces of social isolation in childhood. Journal of Consulting and Clinical Psychology, 56 (6), 916-924. https://doi.org/10.1037/0022-006X.56.6.916

Rubin, K. H., (1982). Non-social play in preschoolers: Necessary evil? Child Development, 53, 651-657.

Rubin, K. H., Bowker, J. C., \& Kennedy, A. E. (2009). Avoiding and withdrawing from the Peer Group. In K. H. Rubin, W. M. Bukowski \& B. Laursen (Eds.), Handbook of peer Interactions, Relationships, and Groups (pp. 303-317). New York, NY: Guilford.

Rubin, K. H., Coplan, R. J., \& Bowker, J. C. (2009). Social withdrawal in childhood. Annual Reviews of Psychology, 60, 141-171. https://doi.org/10.1146/annurev.psych.60.110707.163642

Rubin, K. H., Hymel, S., \& Mills, R. S. (1989). Sociability and social withdrawal in childhood: Stability and outcomes. Journal of Personality, 57(2), 237-255. https://doi.org/10.1111/j.14676494.1989.tb00482.x

Historial do artigo

Recebido $\quad 10 / 05 / 2016$

Aceite 28/04/2017

Publicado $\quad 07 / 2017$ 
Copyright of Psicologia: Revista da Associacao Portuguesa Psicologia is the property of Associacao Portuguesa de Psicologia and its content may not be copied or emailed to multiple sites or posted to a listserv without the copyright holder's express written permission.

However, users may print, download, or email articles for individual use. 